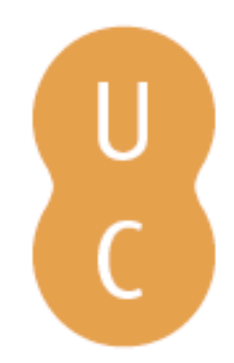

\title{
pompalina
}

\section{Tyche, Kairos et Kronos dans le Phocion de Plutarque}

Autor(es): Leão, Delfim F.

Publicado por: Centro de Estudos Clássicos e Humanísticos; Imprensa da Universidade

URL

persistente: URI:http://hdl.handle.net/10316.2/31672

DOI: $\quad$ DOI:http://dx.doi.org/10.14195/978-989-8281-53-1_13

Accessed : $\quad$ 26-Apr-2023 11:19:46

A navegação consulta e descarregamento dos títulos inseridos nas Bibliotecas Digitais UC Digitalis, UC Pombalina e UC Impactum, pressupõem a aceitação plena e sem reservas dos Termos e Condições de Uso destas Bibliotecas Digitais, disponíveis em https://digitalis.uc.pt/pt-pt/termos.

Conforme exposto nos referidos Termos e Condições de Uso, o descarregamento de títulos de acesso restrito requer uma licença válida de autorização devendo o utilizador aceder ao(s) documento(s) a partir de um endereço de IP da instituição detentora da supramencionada licença.

Ao utilizador é apenas permitido o descarregamento para uso pessoal, pelo que o emprego do(s) título(s) descarregado(s) para outro fim, designadamente comercial, carece de autorização do respetivo autor ou editor da obra.

Na medida em que todas as obras da UC Digitalis se encontram protegidas pelo Código do Direito de Autor e Direitos Conexos e demais legislação aplicável, toda a cópia, parcial ou total, deste documento, nos casos em que é legalmente admitida, deverá conter ou fazer-se acompanhar por este aviso.

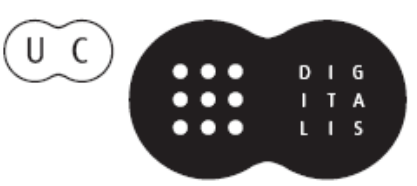




\section{Tychè et Pronoia}

\section{La marche du monde selon Plutarque}

Françoise Frazier et Delfim F. Leão (eds.)

IMPRENSA DA UNIVERSIDADE DE COIMBRA
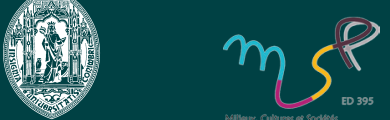


\title{
Tyche, Kairos et Chronos dans le Phocion de Plutarque *
}

\author{
Delfim F. Leão \\ Université de Coimbra
}

\begin{abstract}
Although the terminology employed by Plutarch to express divine influence in human affaires varies a lot, the words tyche, daimon and theos are those that he uses most frequently. In this paper, special attention is given to the action of tyche and other related concepts, precisely because they are attached mainly to fluid and unpredictable factors.

The analysis focuses on the Life of Phocion, which is especially suitable for studying the way the power of divinity is exerted. Phocion had a good character as well as enjoying the benefits of a fine paideia, but despite these important qualities, he was neither capable of preventing Athens from being occupied by the Macedonian forces nor was he able to avoid his own capital punishment. Plutarch does not fail to point out that all this happens because Phocion did not live in a favourable time, and could therefore not exploit completely his own virtues. This notion of a 'favourable time' is explored in order to combine the concepts of kairos and chronos with that of tyche, as a means of justifying Phocion's failures.
\end{abstract}

\section{Remarques préliminaires}

Lorsque les Vitae sont considérées dans leur ensemble, nous pouvons constater que 1'intervention de la Providence divine n'est pas distribuée de façon uniforme, et présente même, à première vue, un tableau assez discordant : en ce qui concerne la partie grecque, l'intervention divine est plus sensible dans les biographies de figures qui ont vécu durant la phase de " décadence " de l'histoire de la Grèce car elle semble être un processus d'une certaine façon irréversible, malgré l'effort d'hommes d'État remarquables comme Phocion; avec Rome, au contraire, la participation de la Providence est plus régulière et apparaît dès la fondation de la ville jusqu'à l'instauration du Principat, fait qui pourrait ne pas être étranger à la perception de l'extraordinaire ascension romaine, dont le succès semblait dû, au moins en partie, à une faveur divine spéciale et durable ${ }^{1}$.

La terminologie utilisée pour exprimer l'influence divine dans les affaires humaines connaît de nombreuses variantes, même si les termes theos, daimon et tyche sont les plus récurrents ${ }^{5}$. Dans cet article, nous ne retiendrons que l'action attribuée à la tyche (et les termes étymologiquement liés), précisément parce que ce concept renvoie, tout particulièrement, à l'action de facteurs à la nature plus fluide et imprévisible. La discussion portera sur la Vita de Phocion, une

* Je remercie Françoise Frazier d'avoir eu la gentillesse de relire la version française du manuscrit.

1 Vide Swain (1989) 280. La contribution d’A. Pérez Jiménez pour ce volume [pp. 169-181] souligne le fait que les héros fondateurs et les premiers grands législateurs ont pu également compter, de façon implicite ou explicite, avec la collaboration divine pour mener à bien leurs projets de stabilisation de la société. Des figures comme Thésée, Romulus, Lycurgue, Numa, Solon et Publicola entrent dans ce champ.

${ }^{2}$ Pour les termes qui renvoient à la Providence divine, vide la liste présentée par Swain (1989) 298-302. 
figure qui, en comparaison avec d'autres hommes d'État, a été relativement négligée par les spécialistes de Plutarque. Pourtant l'existence de Phocion semble réunir des conditions particulièrement favorables pour contrebalancer l'action de la tyche: en effet, Phocion possédait de bonnes qualités de caractère, auxquelles s'ajoutait une éducation soignée, conjonction qui aurait pu en faire un personnage exceptionnel. C'est ainsi qu'il a été nommé général quarante-cinq fois, un nombre sans précédent, que ce soit parmi ses contemporains ou parmi les hommes d'État qui l'ont précédé3. Malgré cela, il n'a réussi ni à empêcher que la ville soit occupée par les forces macédoniennes aux ordres d'Antipatros, ni à échapper à la disgrâce de la peine capitale. Cela est survenu, précisément, parce que Phocion n'a pas vécu durant le temps adéquat pour faire fructifier ses qualités et en tirer le meilleur parti. Ainsi il faut prendre en compte les notions d' "occasion ", kairos, et de « temps chronologique » (marqué par le fil des générations), chronos, conjuguées avec la tyche pour expliquer le malheur de Phocion dans son action publique, association que l'on trouve déjà chez Platon 4 .

\section{Tychelfortune}

Il est hors de question de discuter où même d'esquisser, dans les limites de ce travail, la complexité du terme tyche (et de l'équivalant latin fortuna). En effet, bien que tyche ne soit pas mentionnée par Homère, elle apparaît déjà chez Hésiode (Th. 360), comme une des Océanides, et sa présence s'affirme dans la littérature postérieure. Outre cette abondance de témoignages, qui rendent plus difficile une compréhension globale de son domaine d'action, il faut aussi être conscient du fait que le terme tyche n'a pas toujours les mêmes implications sur l'existence humaine. Si l'on se réfère à l'étymologie, la connexion de tyche avec

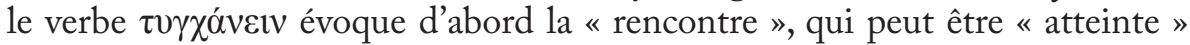
du succès mais aussi heurt contre des obstacles, ou encore, de façon plus neutre, « coïncidence » avec telle ou telle circonstance 5 . Enfin, étant donné que Plutarque est un Grec qui a vécu sous l'empire romain, il semble pertinent de commencer par quelques remarques sur la relation possible entre les concepts de tyche et de fortuna durant le Principat. Le culte populaire de la fortuna dans le monde romain est très ancien. Étymologiquement lié à ferre, le mot signifierait à l'origine 'quelqu'un qui apporte', donc une divinité favorable 'qui apporte la

${ }^{3}$ Cf. Plutarque, Phoc. 8.1-2. Bien que cette information soit généralement admise, Bearzot (1993), 124-126, doute de son exactitude historique, parce qu'elle ne trouve pas de confirmation dans les autres sources. Comme on le verra plus tard, il s'agit probablement d'une exagération de Plutarque destinée à renforcer le caractère supérieur de Phocion.

${ }^{4}$ Platon, dans un passage des Lois (4.709b7-8) soutient que la divinité (theos) contrôle tout, mais que la tyche et le kairos peuvent collaborer avec la divinité dans la conduite des affaires humaines. À ces facteurs, Platon joint encore l'intervention d'un quatrième facteur: 1'art (techne). La référence à techne n'a pas un parallèle direct chez Plutarque, mais peut être assimilée à l'importance attribuée à la paideia, comme nous le verrons plus loin, d'autant plus que, dans le cas de Phocion, l'éducation fut reçue à l'Académie (cf. infra commentaire à Phoc. 4.2). Je remercie Françoise Frazier d'avoir eu la gentillesse de $m$ 'indiquer ce passage très intéressant de Platon.

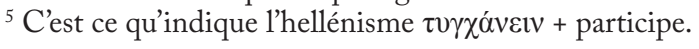


bonne chance, le succès', un esprit protecteur des hommes et des lieux, apparenté au genius ${ }^{6}$. Dans la littérature, néanmoins, l'éventail des significations était plus vaste et assumait, encore une fois, des idées contradictoires. Cette réalité s'est accentuée au fur et à mesure que l'influence de la tyche grecque, en tant que personnification de la fortuna caeca, s'est superposée aux notions romaines initiales, préférentiellement positives. En effet, durant la période républicaine, la notion de fortunaltyche était encore peu fréquente chez les auteurs latins. Son importance grandit dans la production littéraire de l'époque d'Auguste, mais ce n'est que lors de la pleine période impériale qu élle devint, de fait, une entité importante ${ }^{7}$. Or, c'est précisément la réalité de ce moment qui est la plus intéressante, étant donné qu'elle correspond au temps de Plutarque ${ }^{8}$. Pline l'Ancien, qui fut contemporain du biographe de Chéronée, rapporte, dans un passage particulièrement expressif, 1'omnipotence de cette divinité capricieuse qui gagnait de plus en plus de terrain sur le panthéon olympique traditionnel ${ }^{9}$. Il conviendrait pour cela d'évoquer une partie des réflexions qu'il dédie à la nature de la fortuna, qui se présente comme conditionnant toutes les actions humaines et qui devient objet de toutes ses attentions, au point d'assumer le rôle même de Dieu (N.H. 2.22):

Toto quippe mundo et omnibus locis omnibusque horis, omnium uocibus Fortuna sola inuocatur ac nominatur, una accusatur, rea una agitur, una cogitatur, sola laudatur, sola arguitur et cum conuiciis colitur, uolu<cris uolu>bilisque, a plerisque uero et caeca existimata, uaga, inconstans, incerta, uaria indignorumque fautrix. Huic omnia expensa, buic feruntur accepta, et in tota ratione mortalium sola utramque paginam facit, adeoque obnoxiae sumus sortis, ut ipsa pro deo sit qua deus probatur incertus.

De fait dans le monde entier, en tout lieu, à toute heure les voix de tous les hommes invoquent et nomment la seule Fortune; on n'accuse qu'elle, elle seule est coupable, on ne pense qu'à elle, à elle seule vont les éloges, les reproches, et on l'adore en l'insultant ; ailée et volage, regardée même comme aveugle par la plupart, vagabonde, inconstante, incertaine, changeante, elle favorise ceux qui n'en sont pas dignes. On lui impute tout le passif et tout l'actif ; sur le grand livre de comptes de l'humanité, elle seule remplit les deux colonnes, et notre condition est si soumise que la Fortune même, qui prouve l'incertitude de Dieu, prend la place de Dieu ${ }^{10}$.

Les caractéristiques de la fortuna évoquées ici correspondent, en effet, à l'importance qui lui est accordée dans des œuvres comme le Satiricon de

${ }^{6}$ Kajanto (1981) 505 et 521.

${ }^{7}$ Vide Kajanto (1981) 533, 538 et 542, respectivement.

${ }^{8}$ Même en reconnaissant qu'on ne peut pas tout simplement appliquer à Plutarque ce qu'on dit d'autres auteurs qui ont vécu dans la même époque que lui. L'idée c'est présenter seulement un parallèle qui nous semble être très suggestif.

${ }_{9}^{9}$ Une importance qu'elle partageait, du reste, avec l'attention dédiée aux cultes orientaux et au christianisme émergent.

${ }^{10}$ Texte établi et traduit par Jean Beaujeu, Pline l'Ancien - Histoire Naturelle. Livre II (Paris, 1950). 
Pétrone ou l'Âne d'or d'Apulée (comparable à celle de la tyche dans le roman grec), où cette divinité apparaît comme la réelle instigatrice de 1'action ${ }^{11}$. Il a déjà été également souligné que les caractéristiques littéraires de la fortuna étaient, à cette époque, pratiquement communes à celle de la tyche hellénistique. Cette réalité était motivée, du moins en partie, par certaines conditions historiques, que Kajanto ${ }^{12}$ synthétise de la façon suivante : "Tyche was a dominant figure in the literature of the Hellenistic period. Two factors contributed to her popularity. The Olympic gods were rapidly losing ground. This created a religious vacuum, which was in part filled by Tyche. Again, the time of Alexander the Great and of the Epigoni was a period of great upheavals. Many great states, e. g. the Persian Empire, fell to pieces, and new ones were founded instead. The success of an individual seemed often to depend more upon chance than upon his own efforts. This may explain why fickle Tyche, the personification of blind chance, gained so important a position in men's minds. "Cette proximité entre la notion de fortuna et celle de la tyche hellénique ${ }^{13}$, ainsi que le fait que Phocion ait vécu précisément à une époque où commençaient à se faire sentir les effets des grands changements évoqués dans le passage de Kajanto, aide à comprendre l'importance de la tyche dans la biographie de Phocion. C'est l'application pratique de ce point concret que nous allons maintenant examiner dans le cas spécifique de Phocion ${ }^{14}$.

\section{La Vita de Phocion}

La Vie de Phocion présente, au départ, des conditions exceptionnelles pour étudier l'interaction de concepts de tyche, kairos et chronos, puisque l'existence de Phocion (qui vécut entre 402 et 318 avant J.C.) a coïncidé avec la progressive décadence politique et militaire d'Athènes, parallèle à l'irrésistible ascension de la Macédoine (avec Philippe et Alexandre), et connu les incertitudes des premières années des Diadoques. Du reste, la façon dont Plutarque ouvre la biographie, en comparant l'action de Démade et de Phocion, le marque nettement (Phoc. 1.1-6) :

${ }^{11}$ Au sujet de cette réalité dans le Satiricon de Pétrone, vide Leão (1998) 119-131. Du reste, du point de vue de la structure narrative, l'intervention d'une divinité comme la Fortuna est très utile, puisqu'elle permet d'imprimer du dynamisme et de la variation au rythme discursif. Mais il est pertinent de remarquer que la Fortune pouvait aussi fonctionner comme un agent indirect d'une divinité plus haute, comme est le cas de son l'action dans l'Âne d'or d'Apulée (lui même un platonicien), à l'égard de l'affirmation de la puissance d'Isis et Osiris (e.g. 11.15.1-3).

12 (1981) 527-528.

${ }^{13}$ Sur la nature de la tyche hellénistique, voir aussi le célèbre passage de Polybe (citant Démétrios de Phalère), 29.21, et les réflexions de Marie-Rose Guelfucci dans ce même volume, sur la relation entre tyche, la marche de l'histoire et les politeiai [pp. 162-165].

${ }^{14}$ Une lecture plus vaste de l'utilisation du concept de tyche dans l'œuvre de Plutarque est hors de nos objectifs. De toute façon, il nous semble difficile trouver une définition et un domaine d'application qui soient également valables pour les Moralia et pour les Vitae. Sur les difficultés que pose cette notion, vide l'introduction générale de F. Frazier [pp. III-XXIII]. 


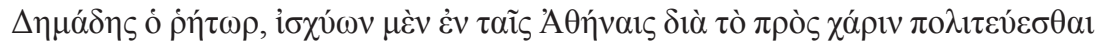

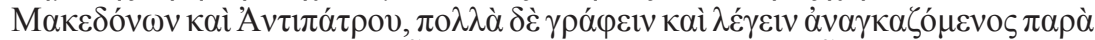

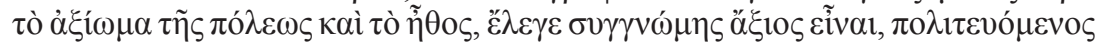

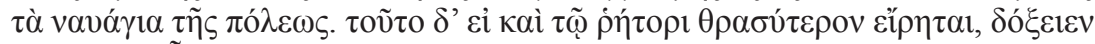

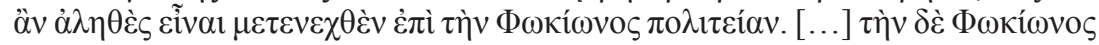

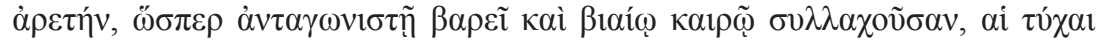

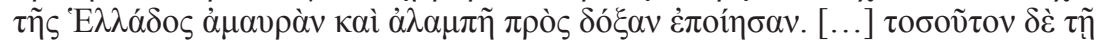

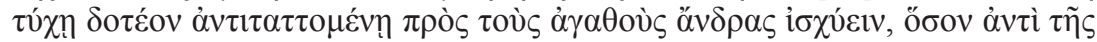

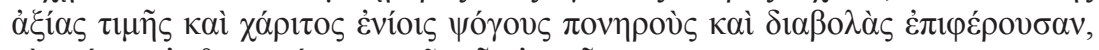

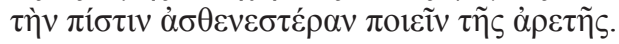

L'orateur Démade, dont la grande influence à Athènes provenait de sa politique de complaisance à l'égard des Macédoniens et d'Antipatros, étant contraint de faire beaucoup de discours et de propositions contraires à la dignité et au caractère de la ville, disait: «Je mérite le pardon parce que j’administre les débris du naufrage de la cité. » Cette parole, si elle paraissait pleine d'effronterie dans la bouche de Démade, pourrait bien être vraie, appliquée au gouvernement de Phocion. [...] Tout au contraire, la vertu de Phocion, par un effet du sort, eut à se mesurer à des circonstances pénibles et cruelles, au point d'être éclipsée et privée de l'éclat de la gloire par les malheurs de la Grèce. [...] Tout ce qu'il faut accorder à la Fortune, quand elle s'attaque aux hommes de coeur, c'est qu'elle attire à quelques-uns d'entre eux, au lieu de la reconnaissance et de l'honneur qui leur sont dus, de méchantes critiques et des calomnies, qui font paraître plus faible leur réputation de vertu ${ }^{15}$.

Même si l'on admet que Plutarque puisse être trop sévère avec Démade, dans la mesure où cet orateur avait en effet rendu de nombreux services à la cité $\mathrm{d}^{\prime}$ Athènes ${ }^{16}$, il est important de souligner, dans cette ouverture, 1'idée exprimée à travers l'allégorie bien connue du vaisseau de 1'État — que la ville

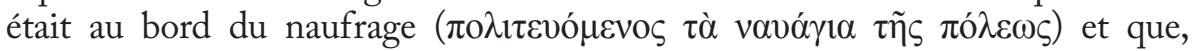
dans ces circonstances, il était difficile de faire plus que parer aux urgences qui survenaient ${ }^{17}$. Et si le biographe répugne à reconnaître la pertinence du jugement en ce qui concerne Démade, il l'admet sans réserve quand il est appliqué à Phocion : ses qualités intrinsèques (arete) n'ont pu se développer pleinement,

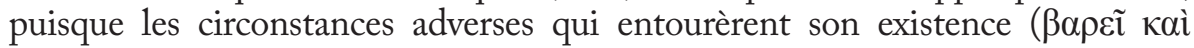

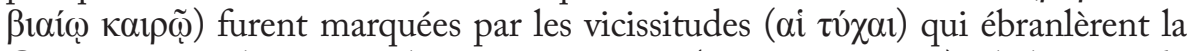
Grèce et empêchèrent qu'il eût une notoriété (doxa, time, charis) à la hauteur de son mérite et obtînt le crédit et la confiance (pistis) qui auraient dû lui revenir. Dès l'ouverture de la Vita, Plutarque marque bien que l'arete ne suffit pas pour garantir le succès escompté, puisqu il faut compter avec la présence de la tyche $e^{18}$

15 Texte établi et traduit par Robert Flacelière et Émile Chambry, Plutarque. Vies. Tome X. Phocion - Caton le Jeune (Paris, 1976).

${ }^{16}$ Qui auraient justifié que lui soit dédiée une statue dans l'agora (cf. Dinarque, 1.101) ; sur son action, voir l'étude de Brun (2000).

${ }^{17} \mathrm{Cf}$. Praec. ger. reip. 803a. On détecte ici une influence très proche du Politique de Platon (e.g. 272e-273e, 296e-297a, 298a).

${ }^{18}$ Ici comprise comme 'infortune' ou 'disgrâce', lorsque, au pluriel, elle désigne les vicissitudes 
et du kairos (« l'occasion »), qui peuvent empêcher les potentialités d'un caractère d'exception de se réaliser, de donner toute leur mesure ${ }^{19}$. Cette idée est reprise et amplifiée dans le chapitre suivant de la biographie, qui souligne la relation entre bybris, tyche et le gouvernement de la polis.

Après cette considération plus théorique, Plutarque reprend le tableau spécifique des Vitae, en introduisant la comparaison entre les circonstances qui entourèrent l'action politique du Romain, Caton le Jeune, où les mêmes principes se trouvent opérants (Phoc. 3.2-5):

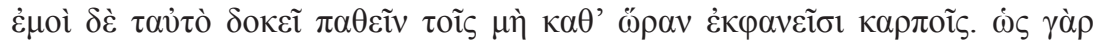

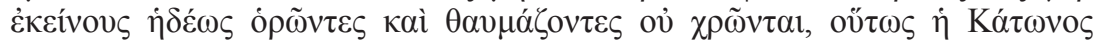

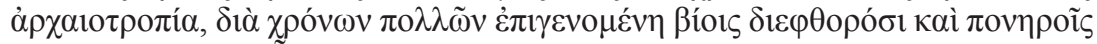

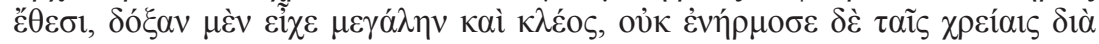

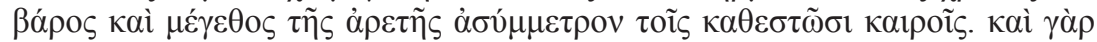

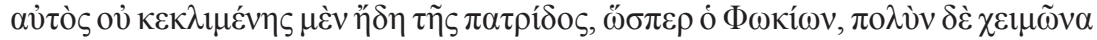

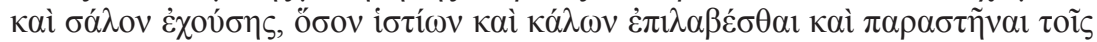

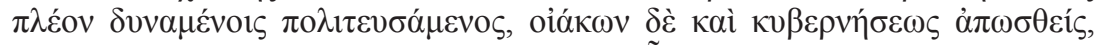

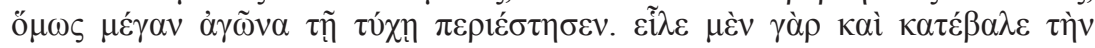

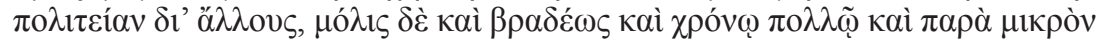

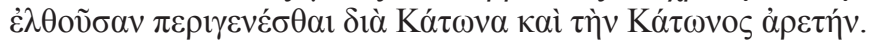

Il lui advint, selon moi, ce qui arrive aux fruits venus hors saison: on les voit avec plaisir, on les admire, mais on n'en use pas. De même, les mœurs antiques de Caton, apparaissant après plusieurs siècles dans une société corrompue et dépravée, lui valurent beaucoup de renommée et de gloire, mais elles nétaient pas ajustées aux nécessités de la politique, à cause de l'austérité et de la grandeur de sa vertu, disproportionnées par rapport à lépoque où il vivait. En fait, lorsque parut Caton, sa patrie ne penchait pas encore vers la ruine, comme celle de Phocion, mais elle était agitée par une grande tempête, et, dans la direction du vaisseau de l'État, il mit seulement la main aux voiles et aux cordages à côté d'hommes plus puissants que lui, et il fut écarté du gouvernail et de la barre, ce qui d'ailleurs ne l'empêcha de livrer un grand combat contre la Fortune. Si celle-ci renversa et perdit la République grâce à d'autres que lui, ce fut à grand-peine, lentement et après de longs efforts, et encore s'en fallut-il de peu que la République ne triomphât grâce à Caton et à la vertu de Caton.

Il a déjà été signalé que, même si les Vitae de Phocion et de Caton appartiennent au groupe des quatre paires de biographies auxquelles manquent

(tychai) qui marquaient la décadence de la Grèce, et comme 'chance' ou 'fortune', à la fin du passage cité, qui a une application plus générale. Dans le commentaire à ce passage, Duff (1999), 137 , identifie l'action de la tyche avec " fate or the will of the gods ", une idée qu'il avait déjà exprimée un peu avant (p. 123), quand il affirma « often in the Lives tyche means either chance or providence ». Même si on a des difficultés à souscrire immédiatement à cette déclaration, elle souligne néanmoins le fait que, dans les Vitae, tyche et Providence ne sont pas nécessairement opposées.

${ }_{19}$ Bearzot (1993), 123-124, considère que la présentation de Phocion est une création littéraire trop positive pour être réelle, et relève les contradictions et exagérations dérivées des intentions apologétiques de Plutarque. 
la synkrisis finale ${ }^{20}$, en réalité, la comparaison n'est pas réellement supprimée, mais plutôt anticipée au commencement du récit, invitant ainsi le lecteur à établir dès le début des parallèles et dont la portée réelle ne sera confirmée que plus tard ${ }^{21}$. Ici, cependant, il est plus important de souligner que Plutarque ouvre ses considérations au sujet de Caton avec le recours à une métaphore agricole pour renforcer à nouveau le poids déterminant qu'a la notion d'occasion opportune. En effet, quand les fruits mûrissent en dehors du temps adéquat ( $\mu$ خे $\left.\kappa \alpha \theta^{\prime} \omega \check{\rho} \alpha \nu\right)$, ils stimulent le plaisir et l'admiration, mais finissent par ne pas remplir la fonction pour laquelle ils ont été créés. De même, la dureté archaïque de Caton a traversé plusieurs générations ( $\delta 1 \grave{\alpha} \chi \rho o ́ v \omega v \pi \mathrm{o} \lambda \lambda \tilde{\omega} v$ ) pour fleurir à une époque qui ne s'accordait plus avec le dépouillement qu'il incarnait. Et même si son action lui apportait une grande renommée ( $\delta$ ó $\xi \alpha v$

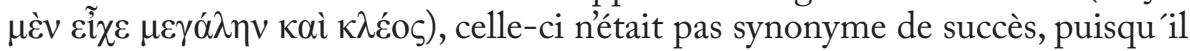
ne réussissait pas à faire adopter ses pratiques par les autres. Et il en était ainsi parce que son arete, si remarquable fût-elle, apparaissait comme 'inadaptée' aux

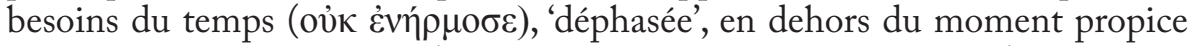

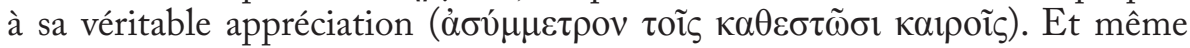
si la situation de Rome au temps de Caton n'était pas comparable à celle d'Athènes - puisque la première bravait seulement une tempête alors que la seconde était entrée dans une décadence irréversible -, il s'est également engagé dans le sauvetage du vaisseau de l'État, menant une grande lutte contre

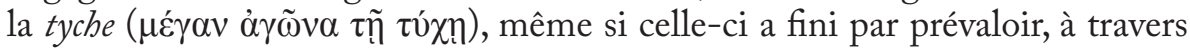
la durée du temps $(\chi \rho o ́ v \omega \pi \mathrm{o} \lambda \lambda \tilde{\omega})$. Ce passage est particulièrement éclairant, puisqu il révèle la façon dont la tyche agit, en articulation avec la notion de kairos et de chronos: en effet, ici, plus qu'un simple hasard ou circonstance, tyche semble correspondre à une action avec des effets à long terme et dont la vraie dimension n'est révélée qu'à travers le temps. Au delà de "l'occasion ", ou 'temps opportun'(kairos), la tyche se sert du chronos (le 'temps chronologique' visible au fil des générations et dans la lenteur de son inexorable action) pour atteindre ses objectifs, ne permettant pas que l'arete de personnes de valeur soit appréciée dans toute sa dimension ${ }^{22}$.

Ce tableau d'une tyche qui combat l'arete d'hommes valeureux est encore plus accentué si l'on prend en compte que Phocion a pu acquérir une paideia du plus haut niveau, à l'Académie, où il a eu l'opportunité de suivre les leçons de Platon et de Xénocrate, qui ont réveillé en lui, dès le début, le désir de rivaliser

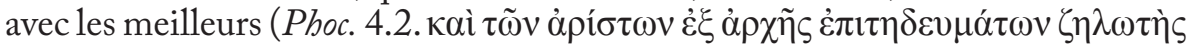
$\gamma \varepsilon v \varepsilon ́ \sigma \theta \alpha \imath)^{23}$. La conjugaison de bonnes qualités innées avec une éducation

${ }^{20}$ Les autres paires sont Thémistocle et Camille, Pyrrhus et Marius, Alexandre et César.

${ }^{21}$ Comme le rappela de façon opportune Trapp (1999) 487-488.

${ }^{22}$ Comme le remarque Tritle (1992), 42-67, l'excellence de Phocion et de Caton émane de leur archaiotropia, de leurs manières démodées, qui, malgré le fait qu'elles renvoient à une arete intemporelle, se révèlent incompatibles avec les circonstances politiques du temps où tous deux vécurent.

${ }^{23}$ Il est souligné, avec pertinence, que ce lien avec l'Académie prépare également le rapprochement entre la figure de ces hommes d'État et celle de Socrate, visible en particulier dans les circonstances qui entourèrent leur mort. Vide Alcalde Martín (1999) 161-163; Trapp (1999) 488. 
soignée commence par donner ses fruits, dès les premiers succès que le jeune Phocion connut, sous les ordres de Chabrias, où, selon Plutarque, il aurait eu un rôle décisif dans la victoire de Naxos. Si ce détail biographique, dont l'authenticité suscite quelques réserves ${ }^{24}$, n'a qu'une importance relative, il faut néanmoins souligner que le triomphe coïncida avec un moment hautement symbolique pour Athènes (Phoc. 6.5-7):

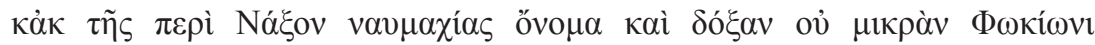

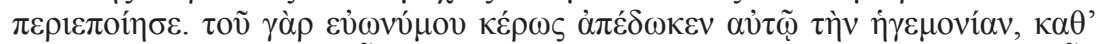

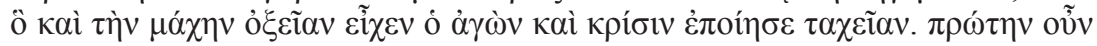

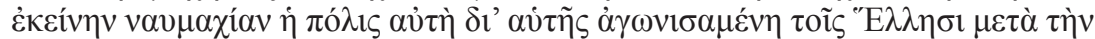

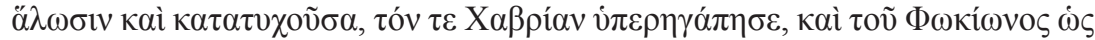

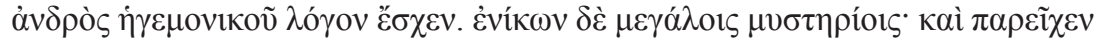

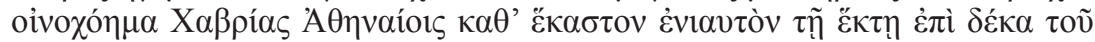

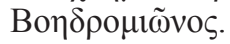

A la bataille navale de Naxos, en particulier, il procura à Phocion une renommée et une gloire éclatantes, en lui donnant le commandement de l'aile gauche, où la lutte fut vive et la décision rapide. Ce fut le premier combat qu'Athènes soutint sur mer contre les Grecs avec ses seules forces après la prise de la ville; ce succès redoubla l'affection des Athéniens pour Chabrias et leur fit concevoir de l'estime pour Phocion, qu'ils jugèrent capable de commander en chef. La victoire de Naxos eut lieu pendant la célébration des Grands Mystères ; c'est pourquoi Chabrias faisait chaque année, le seize de Boédromion, une distribution de vin aux Athéniens.

Le biographe renforce la singularité du moment avec deux aspects notables. La victoire obtenue au large de Naxos était le premier triomphe d'Athènes depuis qu'elle avait été prise par le spartiate Lysandre, dont les troupes avaient démoli les Longs Murs, en 404, au terme de la Guerre du Péloponnèse ${ }^{25}$. Le lien de Phocion avec ce triomphe, dès le début de sa carrière militaire, suggérait qu'il pouvait infléchir l'histoire récente de 1'Attique et permettre à Athènes de récupérer son ancien pouvoir politique et militaire ${ }^{26}$. Un deuxième détail vient souligner cette éventuelle attente :

${ }^{24}$ Diodore (15.34.5) ne mentionne pas Phocion et dit que le commandement de l'aile gauche incombait à Cedon. On ne peut exclure totalement 1'hypothèse que Plutarque ait ajouté cet épisode pour enregistrer un exploit de la jeunesse de Phocion. Bearzot (1993), 127, dit que cette information « ha tutta l'aria di essere l'invenzione di una tradizione apologetica, mirante a sottolineare l'assoluta superiorità di Focione rispetto ai politici ateniese suoi contemporanei e anche predecessori ». Vide aussi Alcalde Martín (2001) 48.

25 Sur les lourdes conditions de la défaite athénienne, vide Hammond (1986) 418 et 488-489.

${ }^{26}$ Cette idée de retour à un passé plus prospère est suggérée aussi par le fait que, plus loin (Phoc. 7.5-6), Plutarque affirme que, en alliant la compétence militaire et oratoire, Phocion se rapprochait des grands politiques d'antan, comme Solon, Aristides et Périclès. En effet, la spécialisation autonome dans ce type de compétences, ainsi que dans le domaine des applications financières, est une des marques de l'époque hellénistique et aussi de la séparation croissante entre le citoyen commun et l'administration de la polis. Au contraire, l'incarnation de l'idéal 
c'est précisément la coïncidence avec la célébration des Mystères d’Éleusis

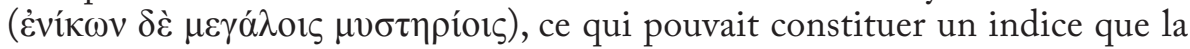
Providence serait favorable à ce projet. Les implications ironiques de cette suggestion voilée s'éclaircissent à la fin de la biographie, lorsque, une fois frustrées les attentes d'un retour de la splendeur passée et après la mort d'Alexandre, Athènes se voit obligée de négocier avec Antipatros les termes de l'accord avec la Macédoine, en ayant recours à nouveau aux services de Phocion. La situation était dure pour Athènes, puisqu'elle prévoyait qu'une garnison macédonienne s'installât en Attique, et serait aggravée par les circonstances où ces changements étaient survenus (Phoc. 28.1-3):

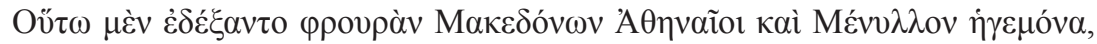

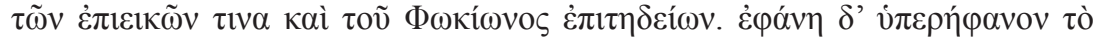

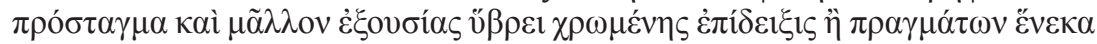

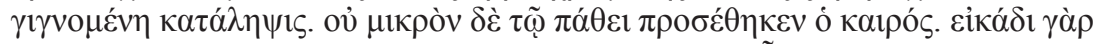

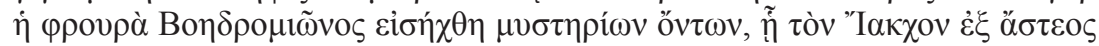

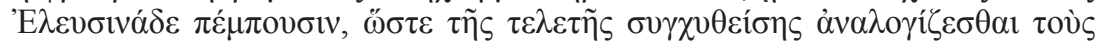

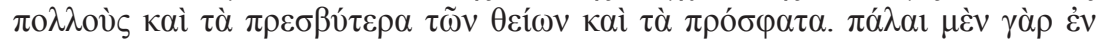

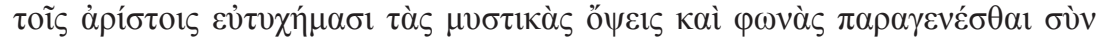

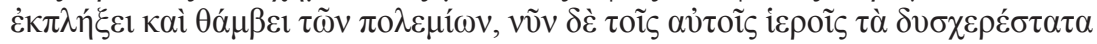

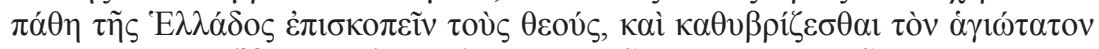

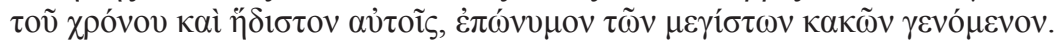

C'est ainsi que les Athéniens reçurent une garnison macédonienne, commandée par Ményllos, homme modéré et ami de Phocion, mais cette exigence d'Antipatros paraissait insultante, et l'on y vit l'étalage d'une autorité fondée sur la violence plutôt qu'une occupation imposée par les circonstances. La date de l'événement accrut beaucoup l'émotion qu'il provoqua: ce fut en effet le vingt de Boédromion que la garnison s'installa, pendant la célébration des mystères, le jour où l'on conduit Iacchos de la ville à Éleusis. En voyant le trouble apporté aux initiations, la plupart des citoyens comparaient les cérémonies présentes à celles d'autres fois : jadis, au temps des grandes victoires, il y avait des apparitions et des voix mystérieuses qui frappaient d'effroi et d'épouvante les ennemis ; maintenant, dans les mêmes solennités, les dieux avaient sous les yeux les plus pénibles malheurs de la Grèce et voyaient profaner les jours les plus saints et les plus joyeux pour eux, marqués désormais par les plus terribles épreuves.

En évoquant la victoire de Naxos (de 376), qui servit de prélude aux succès politiques et militaires de Phocion, Plutarque avait suggéré, sans le dire de façon ouverte, que la coïncidence de cet événement avec la réalisation des Mystères $\mathrm{d}$ 'Éleusis semblait de bon augure pour l'avenir d'Athènes. Cependant, la suite

d'orateur/poète et guerrier (suggéré par l'évocation du frag. 1 Diehl de Archiloque) renvoie à un concept de citoyenneté qui se rapprochait, dans sa formule, de l'idéal nostalgique de la patrios politeia, qui a commencé à se développer à Athènes précisément à la fin de la Guerre du Péloponnèse, tout particulièrement à la suite du désastre de l'expédition en Sicile. À ce sujet, vide Leão (2001) 43-72. 
des événements a exposé la vanité de cet espoir. Et la constatation de cette réalité est présente quand la garnison macédonienne entre à Athènes, près d'un demi-siècle plus tard (en 322), signe éclatant de soumission à Antipatros et de perte réelle de liberté27. La sensation d'un cycle qui s'achève est accentuée par la coïncidence de l'entrée de la garnison dans la ville avec la célébration des Mystères d'Éleusis. Plutarque utilise ici expressément le terme kairos pour souligner toute la tragique ironie du moment. Le terme utilisé généralement pour indiquer l'idée "d'occasion » opportune renforce maintenant le pathos provoqué par la démoralisation des Athéniens, qui comprennent que les dieux

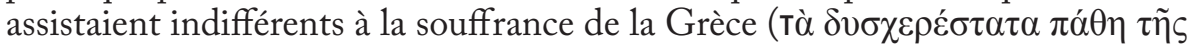

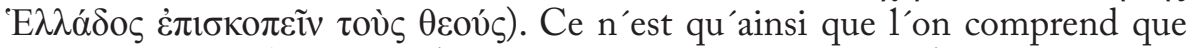
l'acte d'hybris d'Antipatros (deux fois évoqué dans ce passage) puisse entacher le caractère sacré du 'temps' (chronos) de la célébration. Le fait que, dans ce contexte, Plutarque utilise le terme theoi (mais aussi ta theia) pour désigner l'action divine suggère que la décadence de la Grèce correspondait, d'une certaine façon, à un inéluctable dessein supérieur, dont la lecture se révélait finalement dans le long déroulement du 'temps chronologique' (chronos) mais aussi dans le choix symbolique d'un moment spécifique ou 'temps opportun' (kairos) pour rendre évidentes les implications d'une réalité d'une plus grande portée $e^{28}$.

Cet épisode marque, également, le début de la fin politique et biologique de Phocion. Accusé peu après de trahison, l’homme d'État subit les conséquences d'une nouvelle époque marquée par la faillite des idéaux de la polis. La composition de l'assemblée qui prononça sa condamnation à mort témoigne de cette évolution : tous purent y participer, sans distinction de statut ${ }^{29}$. En contraste avec cette décadence généralisée, Phocion présente l'ultime preuve de son arete, en affrontant avec grandeur la mort décidée par cette ville qui lui devait tant. L'exécution coïncide, de nouveau et symboliquement, avec un jour de festivités en 1'honneur de Zeus, ce qui fait que cet assassinat se double d'un crime d'impiété religieuse (Phoc. 37.2:

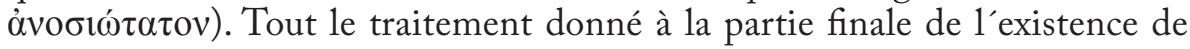
Phocion évoque le jugement de Socrate ${ }^{30}$, que Plutarque place, de façon

${ }^{27}$ En des termes encore plus tragiques que ceux vécus en 404, comme le synthétise de façon suggestive Hammond (1986) 648-649.

${ }^{28}$ Vide Swain (1989) 282.

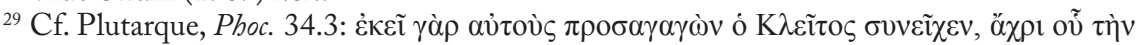

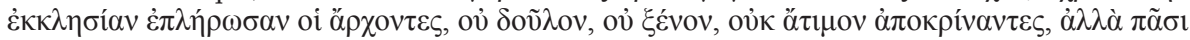

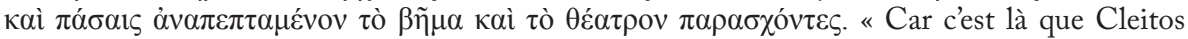
les conduisit et les garda jusqu'à ce que les magistrats eussent réuni l'assemblée. On n'exclut de celle-ci ni les esclaves, ni les étrangers, ni les personnes privées de leurs droits civiques, et on laissa la tribune et le théâtre ouverts à tous et à toutes. » Maria do Céu Fialho, dans sa contribution pour ce volume ("The interplay of textual references in Plutarch's Life of Phocion", p. 202), souligne le fait que le jugement de Phocion est une mascarade démocratique recréée par le dominateur macédonien (Polyperchon) : «For true citizens, the staged trial denounced the farsical democracy that was being acted out, making them aware of danger and demise - the 'shipwreck' of the city, in fact ».

${ }_{30}$ À propos des ressemblances et des différences en ce qui concerne le traitement de la mort 
suggestive, à la fin de la biographie, dans un cadre identique d'irréflexion (Phoc. 38.5):

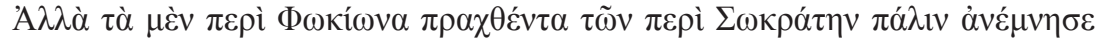

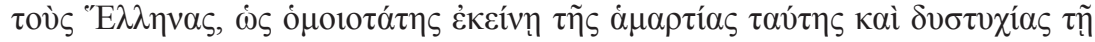

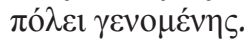

La façon dont périt Phocion rappela aux Grecs la mort de Socrate: ce fut pour la ville une faute et un malheur tout à fait semblables.

L'erreur (hamartia) représentée par cette mort est doublement tragique, puisqu'elle souligne le danger de l'irréflexion collective, ainsi que le gâchis sans gloire d'une des rares sources d'espoir avec laquelle les Athéniens auraient pu compter dans ce moment de grande agitation. Il est également hautement significatif que la biographie se termine sur 1'idée que la mort de Phocion constitue une 'infortune' (dystychia) ou 'mauvaise fortune', dotant ainsi la biographie d'une sorte de composition circulaire : malgré son arete naturelle et son excellente paideia, il lui aurait été nécessaire d'avoir aussi l'appui d'une tyche propice et de vivre dans un temps plus favorable (autant dans le sens de kairos comme dans celui de chronos) afin que ses qualités puissent donner toute leur mesure.

de Socrate, Phocion et Caton (vu que la comparaison est plus favorable à l'homme d'État grec qu'au romain), vide Alcalde Martín (1999); Trapp (1999). Geiger (1999), 358, souligne que, bien que Plutarque mentionne fréquemment Platon et Socrate au cours de son œuvre, ce n'est que dans ce passage des Vitae de Phocion et de Caton que le biographe décrit et discute la mort du philosophe athénien. 


\section{Bibliographie CitÉE}

Alcalde Martín, Carlos, "Rasgos socráticos de la personalidad de Foción en la Vida de Plutarco", in A. Pérez Jiménez, J. García López y Rosa María Aguilar (eds.), Plutarco, Platón y Aristóteles (Madrid, 1999), 159-171.

Alcalde Martín, Carlos, Plutarco. Vida de Foción. Introducción, traducción y notas (Madrid, 2001).

Bearzot, Cinzia, "Introduzione", in Plutarco. Focione e Catone Uticense (Milano, 1993, réimpr. 2001), 91-151.

Brun, Patrice, L'orateur Démade. Essai d'bistoire et d'historiographie (Bordeaux, 2000).

Duff, Timothy E., Plutarch's Lives. Exploring virtue and vice (Oxford, 1999).

Geiger, Joseph, "Plato, Plutarch and the death of Socrates and of Cato", in A. Pérez Jiménez, J. García López y Rosa María Aguilar (eds.), Plutarco, Platón y Aristóteles (Madrid, 1999), 357-364.

Hammond, N. G. L., A History of Greece to 322 B.C. $\left(\right.$ Oxford, $\left.1986^{3}\right)$.

Kajanto, Iiro, "Fortuna” $A N R W$ II.17.1 (1981) 502-558.

LeÃo, Delfim F., As ironias da Fortuna. Sátira e moralidade no Satyricon de Petrónio (Coimbra, 1998).

-, Sólon. Ética e política (Coimbra, 2001).

SwaIn, Simon, "Plutarch: chance, providence, and history", AJPh 110 (1989) 272-302.

Trapp, Michael B., "Socrates, the Phaedo, and the Lives of Phocion and Cato the Younger", in A. Pérez Jiménez, J. García López y Rosa María Aguilar (eds.), Plutarco, Platón y Aristóteles (Madrid, 1999), 487-499.

Tritle, Lawrence A., "Plutarch's 'Life of Phocion': an analysis and critical report”, $A N R W$ II.33.6 (1992) 4258-4297. 